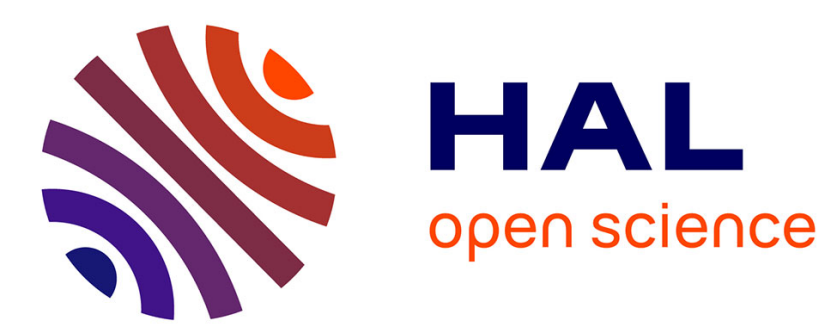

\title{
Quantum Monte Carlo study of the transcorrelated method for correlation factors
}

Hongjun Luo, Wolfgang Hackbusch, Heinz-Juergen Flad

\section{To cite this version:}

Hongjun Luo, Wolfgang Hackbusch, Heinz-Juergen Flad. Quantum Monte Carlo study of the transcorrelated method for correlation factors. Molecular Physics, 2010, 108 (03-04), pp.425-431. 10.1080/00268970903521194. hal-00580677

\section{HAL Id: hal-00580677 https://hal.science/hal-00580677}

Submitted on 29 Mar 2011

HAL is a multi-disciplinary open access archive for the deposit and dissemination of scientific research documents, whether they are published or not. The documents may come from teaching and research institutions in France or abroad, or from public or private research centers.
L'archive ouverte pluridisciplinaire HAL, est destinée au dépôt et à la diffusion de documents scientifiques de niveau recherche, publiés ou non, émanant des établissements d'enseignement et de recherche français ou étrangers, des laboratoires publics ou privés. 


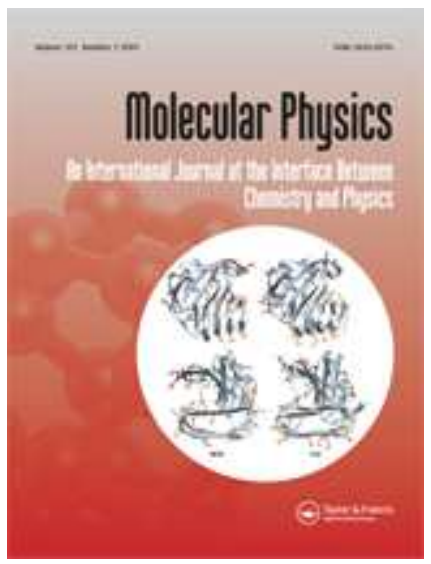

\section{Quantum Monte Carlo study of the transcorrelated method for correlation factors}

\begin{tabular}{|r|l|}
\hline Journal: & Molecular Physics \\
\hline Manuscript ID: & TMPH-2009-0341.R1 \\
\hline Manuscript Type: & Special Issue Paper - In honour of Prof Werner 60th birthday \\
\hline Date Submitted by the \\
Author: & $26-$ Nov-2009 \\
\hline Complete List of Authors: & $\begin{array}{l}\text { Luo, Hongjun; MIS MPG Leipzig, Scientific computing } \\
\text { Hackbusch, Wolfgang; MIS MPG Leipzig } \\
\text { Flad, Heinz-Juergen; TU Berlin, Inst. Math. }\end{array}$ \\
\hline Keywords: & Transcorrelated method, Jastrow factor, Quantum Monte Carlo \\
\hline
\end{tabular}

\section{(s) ScholaroNE" \\ Manuscript Central}




\section{Introduction}

A prevalent prejudice in quantum chemistry is that correlated wave functions of many-electron systems are unwieldy objects which require an enormous number of degrees of freedom for an accurate representation. This point of view originated from large scale configuration interaction (CI) calculations where up to billions of Slater determinants were used in order to achieve accurate results for rather small molecules. A more economical representation of wave functions can be achieved via the exponential ansatz which became popular through its use in coupled cluster (CC) theory. This ansatz incorporates essential physical insights and guarantees size consistency of the method. Another important insight from Hylleraas and collaborators was the astonishing accuracy of expansions in terms of inter-particle distances. A combination of both worlds is the Jastrow ansatz for the wave function

$$
\Psi(\mathbf{R})=e^{\tau(\mathbf{R})} \Phi(\mathbf{R}), \quad \mathbf{R}=\left(\mathbf{r}_{1}, \mathbf{r}_{2}, \cdots, \mathbf{r}_{N}\right),
$$

where $\mathbf{R}$ denotes the combined coordinates of all $N$ particles. The correlation function $\tau$ represents a symmetric function of the electron coordinates and the reference wave function $\Phi(\mathbf{R})$ can be chosen as a single Slater determinant, e.g. from Hartree-Fock, or an appropriate linear combination. Generally, a given ansatz for the reference wave function has an inherent error in the nodal structure which cannot be compensated by the Jastrow factor. Therefore, the Jastrow ansatz should be considered only as an approximation to the exact wave function. The remaining, so-called fixed-node error is typically small, i.e., less that $5 \%$ of the correlation energy, but hard to improve in a systematic manner. Jastrow factors have been extensively studied in condensed matter and nuclear physics [1] but rarely considered 
in quantum chemistry. Remarkable exceptions are the transcorrelated (TC) method of Boys and Handy $[2,3,4,5,6,7,8,9,10]$ developed forty years ago and more recent developments in quantum Monte Carlo (QMC) methods $[11,12,13,14]$. Because of the exponential ansatz, the optimisation of the correlation function is a highly nonlinear problem. This can be handled in a variational manner using either QMC $[11,15,16,17]$ or highly sophisticated diagrammatic techniques, like the Fermi hypernetted chain method $[18,19]$. An alternative nonvariational approach is the TC method of Boys and Handy [4]. The basic idea is to transform the formal Schrödinger equation for the Jastrow ansatz

$$
(\hat{H}-E) e^{\tau} \Phi=0
$$

into the form

$$
\left(e^{-\tau} \hat{H} e^{\tau}-E\right) \Phi=0,
$$

where the similarity transformed Hamiltonian gives only rise to quadratic non-linear terms in the correlation function. According to our previous discussion, Eqs. (1.2) and (1.3) are generally not exactly solvable for a given ansatz of the reference wave function $\Phi$. Instead an approximate solution can be envisaged which satisfies

$$
\int_{\mathbb{R}^{3 N}} d^{3 N} R\left(\frac{\partial e^{-\tau} \Phi}{\partial c_{\alpha}}\right)(\hat{H}-E) e^{\tau} \Phi=0
$$

for any free parameter $c_{\alpha}$ of the correlation function $\tau$ or reference wave function $\Phi$. This transcorrelated formulation strongly resembles the projection equations of CC theory. The error of this approach in the wave function and energy has been characterized by Boys and Handy [2, 4] via the least squares errors of the basis $\left\{\partial e^{\tau} \Phi / \partial c_{\alpha}\right\}$ and corresponding projection basis $\left\{\partial e^{-\tau} \Phi / \partial c_{\alpha}\right\}$ with respect to the exact wave function. For actual calculations, the original Eq. (1.4) poses severe technical difficulties concerning the computation of the integrals. Three different approaches have been studied by Boys and Handy to circumvent this problem. The first approach is to replace the projection basis by a computationally simpler CI-type basis consisting of a set of single and double excited Slater determinants. This has been tested for the Ne atom [5] and gave a total energy which is more than 20 mHartree below the "exact" energy. Another approach is to fix the leading order term which gives rise to Kato's cusp condition and to choose a separable ansatz for the optimised part of the correlation factor. The difficult to handle leading order term therefore does not show up as a derivative on the left side of the Hamiltonian in (1.4) which greatly simplifies the computations. Applications to $\mathrm{LiH}$ and the Be atom are reported in Refs. [6] and [7], respectively. The third approach uses a Gaussian type geminal basis set for the correlation factor which enables the computation of all the integrals involved. Interestingly, it has been observed by Handy that the latter approach behaves similar to a variational calculation [9] in contrast to the other two approaches mentioned before. It is fair to say that the TC calculations of Boys and Handy were of amazing accuracy for those days. However, due to obvious limitations of the computing facilities sometimes very small basis sets were taken which might obscure, together with a mixture of different computational approaches, the actual behaviour of the TC method. It is the purpose of the present work to provide unambiguous results which shed light on the performance of the TC method for Jastrow factor optimisation.

The lack of a variational lower bound, generally attributed to the non-hermitian TC Hamiltonian, is usually considered as a severe problem of the TC approach [20]. Therefore it was largely abandoned until the revival of the method by the work of Ten-no and collaborators. In their work [21, 22, 23, 24, 25], the correlation factor was kept fixed, e.g., using Gaussian geminals, and the transcorrelated Hamiltonian was treated using perturbation theory or the coupled electron-pair approximation. In this sense Tenno's approach resembles to R12 or F12 methods [20] which improve convergence with the basis set for conventional many-particle methods. Another work along this line is the approach of Umezawa et al. $[26,27,28,29]$ to solve the orbital equations in a self-consistent manner for a single Slater determinant, where the correlation factor is fixed up to a single parameter. Like in the present work, QMC techniques were used to compute the integrals.

Within the present work we want to reconsider the TC method in its original formulation (1.4) using the Hylleraas-like correlation basis introduced by Boys and Handy in terms of polynomials of rational 
expressions in the inter-particle distances. The complicated integrals, which prevented straightforward computations forty years ago, have been calculated with QMC. For comparison we performed variational Monte Carlo (VMC) calculations with TC and energy optimised Jastrow factors. Originally, the TC equations were designed to optimise the reference function, i.e., the orbitals which constitute the Slater determinant, and the correlation function simultaneously. We refrain from optimising the orbitals and consider only the correlation function. With this no indeterminacy appears concerning the one-electron part of the correlation function which required an additional contraction equation in the work of Boys and Handy [3].

The paper is organized as follows: In Section 2 we give a detailed description of the TC equation and discuss its relations with the variational approach. Test calculations for atoms and small molecules are presented in Section 3 where we compare the performance of the TC and variational approach. Finally, in Section 4 we give some concluding remarks.

\section{Transcorrelated versus variational treatment of electron correlation}

For a given reference function $\Phi$, the Jastrow ansatz (1.1) usually does not provide an exact solution for the stationary Schrödinger equation

$$
\hat{H} \Psi=E \Psi
$$

with nonrelativistic Hamiltonian

$$
\hat{H}=-\frac{1}{2} \sum_{i} \nabla_{i}^{2}+\sum_{i} v_{e x t}\left(\mathbf{r}_{i}\right)+\sum_{i>j} \frac{1}{r_{i j}} .
$$

in the Born-Oppenheimer approximation. Atomic units have been used throughout the paper. Instead of the exact solution one can search for the correlation function $\tau(\mathbf{R})$ which minimises the expectation value of the energy

$$
E=\min _{\tau} \frac{\int_{\mathbb{R}^{3 N}} d^{3 N} R e^{\tau} \Phi(\mathbf{R}) \hat{H} e^{\tau} \Phi(\mathbf{R})}{\int_{\mathbb{R}^{3 N}} d^{3 N} R e^{\tau} \Phi(\mathbf{R}) e^{\tau} \Phi(\mathbf{R})} .
$$

Here and in the following we assume all wave functions to be real valued. According to the standard procedure we introduce a basis set for the correlation function

$$
\begin{aligned}
U_{\alpha}^{(1)}(\mathbf{R}) & =\sum_{i} u_{\alpha}^{(1)}\left(\mathbf{r}_{i}\right) \\
U_{\beta}^{(2)}(\mathbf{R}) & =\frac{1}{2} \sum_{i \neq j} u_{\beta}^{(2)}\left(\mathbf{r}_{i}, \mathbf{r}_{j}\right),
\end{aligned}
$$

where $U_{\alpha}^{(k)}$ denotes symmetric $k$-particle basis functions. For notational simplicity we skip the index $k$ in the following discussion. Inserting the expansion of the correlation function

$$
\tau(\mathbf{R})=\sum_{\alpha} c_{\alpha} U_{\alpha}(\mathbf{R})
$$

into (2.3) yields a nonlinear eigenvalue problem

$$
\left\langle U_{\alpha} e^{\tau}(\hat{H}-E) e^{\tau}\right\rangle=0
$$

where

$$
\langle\hat{O}\rangle:=\int_{\mathbb{R}^{3 N}} d^{3 N} R \Phi(\mathbf{R}) \hat{O} \Phi(\mathbf{R})
$$


denotes the expectation value of an operator $\hat{O}$ with respect to the normalized reference wave function.

The variational equation (2.6) can be interpreted as the Schrödinger equation for the Jastrow ansatz projected onto the subspace spanned by $\left\{U_{\alpha} e^{\tau} \Phi\right\}$. This means that Schrödinger's equation is only satisfied in a weak sense with respect to the set of test functions $\left\{U_{\alpha} e^{\tau} \Phi\right\}$ and not on the whole Hilbert space. In contrast to the variational approach, the TC method of Boys and Handy for the correlation factor leads to a weak formulation of the Schrödinger equation of the form

$$
\left\langle U_{\alpha} e^{-\tau}(\hat{H}-E) e^{\tau}\right\rangle=0,
$$

where a set of test functions $\left\{U_{\alpha} e^{-\tau} \Phi\right\}$ has been applied. In fact Eqs. (2.6) and (2.8) are closely related since the test functions $U_{\alpha} e^{\tau} \Phi$ and $U_{\alpha} e^{-\tau} \Phi$ share the same nodal structure. If we consider the limiting case where $\left\{U_{\alpha}\right\}$ forms a complete basis for n-body permutationally symmetric functions, the spaces spanned by $\left\{U_{\alpha} e^{\tau} \Phi\right\}$ and $\left\{U_{\alpha} e^{-\tau} \Phi\right\}$ are identical which means that also Eqs. (2.6) and (2.8) become equivalent.

It is only the kinetic energy part of the Hamiltonian which does not commute with the Jastrow factor. Therefore, Eq. (2.8) can be rewritten as

$$
\left\langle U_{\alpha}\left(\hat{H}+[\hat{H}, \tau]-\frac{1}{2} \sum_{i}\left(\nabla_{i} \tau\right)^{2}-E\right)\right\rangle=0,
$$

and the TC energy is

$$
E=\langle\hat{H}\rangle-\frac{1}{2}\left\langle\sum_{i}\left(\nabla_{i} \tau\right)^{2}\right\rangle .
$$

By constant shifts of the basis functions

$$
\tilde{U}_{\alpha}=U_{\alpha}-\left\langle U_{\alpha}\right\rangle
$$

Eq. (2.9) becomes

$$
\left\langle\tilde{U}_{\alpha}\left(\hat{H}+[\hat{H}, \tau]-\frac{1}{2} \sum_{i}\left(\nabla_{i} \tau\right)^{2}\right)\right\rangle=0,
$$

which is decoupled from the transcorrelated energy (2.10). The non-linear character of Eq. (2.12) requires an iterative solution method. Following Boys and Handy [4], we solve the corresponding linear defect equation

$$
\left\langle\tilde{U}_{\alpha}[\hat{H}, \delta \tau]\right\rangle-\sum_{i}\left\langle\tilde{U}_{\alpha}\left(\nabla_{i} \tau\right) \cdot\left(\nabla_{i} \delta \tau\right)\right\rangle=-\left\langle\tilde{U}_{\alpha}\left(\hat{H}+[\hat{H}, \tau]-\frac{1}{2} \sum_{i}\left(\nabla_{i} \tau\right)^{2}\right)\right\rangle,
$$

and update the correlation function $\tau+\delta \tau \rightarrow \tau$ of the Jastrow factor in an iterative manner until convergence has been achieved.

Some of the integrals in Eq. (2.12) are notoriously difficult to compute and must be treated by elaborate numerical quadrature schemes. In order to simplify the problem Boys and Handy devised an alternative approach where the set of test functions $\left\{U_{\alpha} e^{-\tau} \Phi\right\}$ is replaced by $\left\{e^{-\tau} \Phi_{r}^{\prime \prime}\right\}$ which is more convenient from a computational point of view. Here $\Phi_{r}^{\prime \prime}$ denotes Slater determinants generated via double excitations from the reference determinant $\Phi$. By construction, these test functions, however, have different nodal structures and their span differs from the subspace spanned by $\left\{U_{\alpha} e^{-\tau} \Phi\right\}$. Therefore no basis set limit exits, in the sense discussed in the previous paragraph, such that the TC and variational approaches become equivalent. Furthermore, for the new set of test functions, Eq. (2.12) cannot be rigorously satisfied anymore and has to be solved in the sense of a least squares approximation [5].

Even for present day computing facilities, not to mention the situation faced by Boys and Handy forty years ago, TC calculations with the original set of test functions $\left\{U_{\alpha} e^{-\tau} \Phi\right\}$ are rather challenging. On the one hand basis functions $U_{\alpha}$ are required which provide compact and accurate representations for correlation functions and on the other hand these functions must be also convenient from the computational point of view. In our previous work we studied tensor product wavelets within the framework 
of diagramatic multiresolution analysis [30] in order to meet both requirements. Quite recently we have also considered canonical tensor product approximations for correlation functions [31] as an alternative approach to reduce the computational complexity of the corresponding integrals. In order to be applicable to the TC method both approaches require extensive software development. The main purpose of the present work is to test the accuracy of the original TC equation (2.12) and to perform a direct comparison with variational calculations. Therefore we have chosen QMC techniques for the computation of the integrals. This enables us to use well established basis functions for the Jastrow factor. The expectation value of a general operator $\hat{O}$ can be rewritten as

$$
\langle\hat{O}\rangle=\int_{\mathbb{R}^{3 N}} d^{3 N} R O_{L}(\mathbf{R}) \rho(\mathbf{R}),
$$

with

and measure

$$
O_{L}(\mathbf{R})=\frac{\hat{O} \Phi(\mathbf{R})}{\Phi(\mathbf{R})}
$$

$$
\rho(\mathbf{R})=\frac{|\Phi(\mathbf{R})|^{2}}{\int_{\mathbb{R}^{3 N}} d^{3 N} R|\Phi(\mathbf{R})|^{2}} .
$$

Generating a set of random samples $\left\{\mathbf{R}_{m}\right\}_{m=1, M}$ according to the distribution function $\rho$ from a Metropolis algorithm, the expectation value of $\hat{O}$ can be estimated by

$$
\langle\hat{O}\rangle \approx \frac{1}{M} \sum_{m=1}^{M} O_{L}\left(\mathbf{R}_{m}\right) .
$$

The statistical error of this approximation converges with $\mathcal{O}\left(M^{-\frac{1}{2}}\right)$ independent on the number of particles.

\section{Transcorrelated QMC calculations}

We have performed some test calculations for the ground states of the atoms $\mathrm{Be}, \mathrm{O}, \mathrm{Ne}$ and the molecules $\mathrm{CH}_{4}, \mathrm{H}_{2} \mathrm{O}$. In these calculations, we have used the polynomial basis originally suggested by Boys and Handy [5] which became the standard basis in QMC after its revival by the work of Schmidt and Moskowitz [12]. For the representation of the pair-correlation function this basis is given in terms of polynomials of rational functions in the inter-partical distances, i.e.,

$$
u_{\alpha}^{(2)}\left(\mathbf{r}_{i}, \mathbf{r}_{j}\right)=\left(\bar{r}_{i I}^{m} \bar{r}_{j J}^{n}+\bar{r}_{j I}^{m} \bar{r}_{i J}^{n}\right) \bar{r}_{i j}^{o}
$$

with

$$
\bar{r}_{i I}=\frac{r_{i I}}{1+r_{i I}}, \quad \bar{r}_{i j}=\frac{r_{i j}}{1+r_{i j}},
$$

where $m, n, o$ are taken to be non-negative integers and $I, J$ are indices of nuclei. Such basis functions are characterized by a multi-index $(m, n, o, I, J)$ which is simply denoted by $\alpha$ here and in the following. For atoms, the largest basis used in our calculations contains 49 polynomials. It is simply constructed by taking into account all polynomials (3.1) with $m+n+o \leq 6$. Among these 49 polynomials, there are 6 one body terms (with $m \neq 0, n=o=0$ ) and 43 two body terms to which spin dependent coefficients have been assigned except for He atom. Therefore our atomic basis for the pair-correlation function has in total 92 spin dependent degrees of freedom. Wave functions with spin dependent Jastrow factors may not be eigenstates of the total spin operator $\hat{S}^{2}$. A study by Huang et al. [32], however, revealed that possible spin contaminations are small. For molecules, the multi-index of the basis functions also depends on indices for the nuclei $(I, J)$ which increases their number considerably and requires a more adaptive construction of the basis set. In the case of $\mathrm{H}_{2} \mathrm{O}$ and $\mathrm{CH}_{4}$, basis sets with 103 and 125 spin dependent 


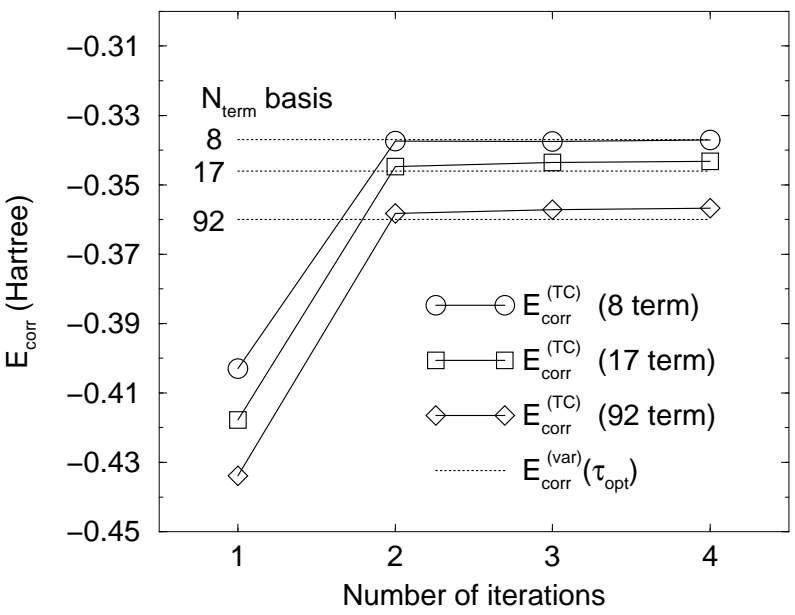

Figure 3.1: Convergence of the TC correlation energy (Hartree) of the Ne atom for different basis sets: (○) 8 term basis of Boys and Handy, ( $\square$ ) 17 term basis of Schmidt and Moskowitz, $(\diamond) 92$ term spin dependent basis. For comparison we have shown the corresponding variational correlation energies for energy optimised correlation functions $\tau_{\text {opt }}$ from first-order iterative Jastrow perturbation theory (dotted lines).

degrees of freedom have been employed in our calculations, respectively. Within the present work, we consider only the TC equation for the correlation factor, and the reference wave functions were taken from Hartree-Fock calculations. For atoms, Slater-type orbitals were taken from Clementi and Roetti [33] and for the molecules Gaussian-type orbitals have been obtained from MOLPRO [34].

As can be expected, the polynomial basis (3.1) leads to ill-conditioned matrices, a problem which is enhanced by statistical fluctuations from the Monte Carlo algorithm. To keep the equations tractable a singular value decomposition (SVD) has been applied to get rid of statistical fluctuations and almost linear dependencies in the basis. The details of our procedure are as follows: usually we take an ensemble of 100 random walkers which undergo random walks of $2 \times 10^{5}$ Monte Carlo steps. The matrix elements for the linear TC equation (2.13), in the following briefly denoted $\mathbf{A} \mathbf{x}=\mathbf{b}$, are calculated for each of the walkers separately, so we have an ensemble of matrices $\mathbf{A}^{(i)}$ and vectors $\mathbf{b}^{(i)}$ where $i$ refers to the index of the walker. Taking an ensemble average yields the matrix $\mathbf{A}$, and vector $\mathbf{b}$. The SVD applied to the matrix A gives

$$
\tilde{\mathbf{A}}=\mathbf{U}^{T} \mathbf{A V}
$$

where $\mathbf{U}$ and $\mathbf{V}$ are orthogonal matrices and $\tilde{\mathbf{A}}$ is a diagonal matrix with non-negative elements (singular values). Thus the linear equation is diagonalized as

$$
\tilde{\mathbf{A}} \tilde{\mathbf{x}}=\tilde{\mathbf{b}}, \quad \tilde{\mathbf{x}}=\mathbf{V}^{T} \mathbf{x}, \quad \tilde{\mathbf{b}}=\mathbf{U}^{T} \mathbf{b} .
$$

In order to get an estimate of the statistical error of the matrix $\tilde{\mathbf{A}}$ and vector $\tilde{\mathbf{b}}$, we performed the transformations (3.3) and (3.4) for each $\mathbf{A}^{(i)}$ and $\mathbf{b}^{(i)}$ separately, and calculated the standard error of the matrix elements of $\tilde{\mathbf{A}}$ and $\tilde{\mathbf{b}}$ based on the fluctuation of $\tilde{\mathbf{A}}^{(i)}$ and $\tilde{\mathbf{b}}^{(i)}$, respectively. By denoting the diagonal matrix elements of $\tilde{\mathbf{A}}$ and their standard errors as $\tilde{A_{\alpha}}$ and $\delta \tilde{A_{\alpha}}$, respectively, the solution of the linear equation simply becomes $\tilde{x}_{\alpha}=\tilde{b}_{\alpha} / \tilde{A}_{\alpha}$. To prevent the fluctuations being amplified by small singular values, we set $\tilde{x}_{\alpha}=0$ if $\left|\delta \tilde{b}_{\alpha}\right|>\tilde{b}_{\alpha}$ or $\left|\delta \tilde{b}_{\alpha}\right|>\tilde{A}_{\alpha}$ or $\left|\delta \tilde{A}_{\alpha}\right|>\tilde{A}_{\alpha}$.

The first test calculations were carried out for the Ne atom. In order to check the dependence of the TC correlation energy on the choice of the basis functions $\left\{U_{\alpha}\right\}$, we have performed calculations for three different basis sets, i.e., the 8 term basis of Boys and Handy (cf. Table 1. in Ref [5]), the 17 term basis of Schmidt and Moskowitz (cf. Table VI. in Ref [12]) and the 92 term spin dependent basis described before. 
Figure 3.2: Comparison of TC and variational correlation energies. Ratios with respect to the corresponding fixed-node DMC correlation energies are shown. The variational energies are calculated with TC correlation factors as well as for energy optimised correlation factors from first-order iterative Jastrow perturbation theory. For atoms the 92 term basis has been used whereas for $\mathrm{H}_{2} \mathrm{O}$ and $\mathrm{CH}_{4}$ the 103 and 125 term basis have been employed, respectively.

The convergence of the iterative solution of the transcorrelated equation (2.12) using (2.13) is shown in Fig. 3.1, where the initial correlation function was set to $\tau=0$. It turned out that the iteration scheme converged very fast, i.e., within two to three iterations for all three basis sets. For comparison, we have also calculated energy optimised correlation functions $\tau_{o p t}$ for the same basis sets using first-order iterative Jastrow perturbation theory [17]. The corresponding variational correlation energies, which are obtained from VMC calculations of the expectation value of the energy, are indicated in Fig. 3.1 as dotted lines. It can be seen that for all three basis sets, the TC and variational correlation energies are in very close agreement to each other.

For our other test systems $\mathrm{Be}, \mathrm{O}, \mathrm{H}_{2} \mathrm{O}$ and $\mathrm{CH}_{4}$, calculations have been only performed with the large spin dependent bases discussed before. It is interesting to calculate besides the TC correlation energies $E_{\text {corr }}^{(T C)}$ from Eq. (2.10) also the variational correlation energy $E_{\text {corr }}^{(v a r)}\left(\tau_{T C}\right)$ for the TC Jastrow factors. The results for TC Jastrow factors have been compared in Table 3.1 with correlation energies for energy optimised Jastrow factors $E_{\text {corr }}^{(v a r)}\left(\tau_{\text {opt }}\right)$. In Fig. 3.2 these correlation energies are shown for our test systems relative to the fixed-node diffusion quantum Monte Carlo (DMC) results $E_{\text {corr }}^{(D M C)}$ which can be considered as benchmarks for variational energies in the complete basis set limit. We obtained very close agreement, i.e., within $2 \%$ for all three types of correlation energies. In particular, we never observed that (2.10) gives an energy significantly lower than the corresponding variational value. The good agreement between transcorrelated and variationally optimised correlation energies suggests that also the corresponding Jastrow factors are of comparable quality.

Our calculations show a similar qualitative behaviour as the Gaussian-type geminal approach of Handy [9] which can be expected since this approach uses the same TC equation (2.12) and differs only in the choice of the basis set for the correlation function. Based on their calculations for the Be atom and $\mathrm{LiH}$ $[6,7]$, it has been argued by Handy [9] that a better convergence behaviour can be expected if the leading order term is not fixed by Kato's cusp condition. We have tested this argument in our calculations and obtained almost the same results independent of whether or not this term was fixed. 
Table 3.1: Transcorrelated and variational correlation energies (mHartree). The variational energies are calculated with VMC for both TC $\left(\tau_{T C}\right)$ and energy optimised correlation functions $\left(\tau_{\text {opt }}\right)$. For comparison, the fixed-node DMC and exact nonrelativistic correlation energies are given.

\begin{tabular}{|c|c|c|c|c|c|c|c|}
\hline & $\mathrm{Be}$ & $\mathrm{O}$ & & $\mathrm{Ne}$ & & $\mathrm{H}_{2} \mathrm{O}$ & $\mathrm{CH}_{4}$ \\
\hline$N_{\text {term }}$ & 92 & 92 & 8 & 17 & 92 & 103 & 125 \\
\hline TC & $-0.0769(2)$ & $-0.2253(2)$ & $-0.3370(4)$ & $-0.3431(4)$ & $-0.3567(3)$ & $-0.3290(5)$ & $-0.2634(3)$ \\
\hline $\operatorname{VMC}\left(\tau_{T C}\right)$ & $-0.0772(2)$ & $-0.2260(5)$ & $-0.3367(5)$ & $-0.3446(3)$ & $-0.3581(2)$ & $-0.3310(4)$ & $-0.2657(2)$ \\
\hline $\operatorname{VMC}\left(\tau_{o p t}\right)$ & $-0.0778(2)$ & $-0.2268(4)$ & $-0.3369(5)$ & $-0.3458(4)$ & $-0.3599(5)$ & $-0.331(1)$ & $-0.2678(5)$ \\
\hline DMC & $-0.08415(7)^{a}$ & $-0.2422(5)^{a}$ & & $-0.3752(3)^{a}$ & & $-0.352(1)^{c}$ & $-0.283(1)^{c}$ \\
\hline Exact & $-0.09434^{b}$ & $-0.2579^{b}$ & $-0.3905^{b}$ & & & $-0.3719^{c}$ & $-0.296^{c}$ \\
\hline
\end{tabular}

${ }^{a}$ Ref. [35], ${ }^{b}$ Ref. [36], ${ }^{c}$ Ref. [37]

\section{Conclusion}

Test calculations on several atomic and molecular systems demonstrate that the performance of the original TC equation (2.12) is very close to variational calculations for the determination of correlation functions. This important fact was somewhat obscured by the calculations of Boys and Handy owing to technical limitations and related further approximations. Our work together with the results of Ten-no et al. and Umezawa et al. suggests that the TC method is an interesting alternative to conventional F12 methods where the correlation function is kept fixed throughout the calculation. QMC offers an efficient approach to compute complicated integrals which are otherwise difficult to access.

\section{References}

[1] Clark, J. W., 1979, Progress in Nuclear and Particle Physics Vol. 2, Ed. Wilkinson, D. H., (Pergamon, Oxford), p. 89.

[2] Boys, S. F., 1969, Proc. R. Soc. London Ser. A 309, 195.

[3] Boys, S. F., and Handy, N. C., 1969, Proc. R. Soc. London Ser. A 309, 209.

[4] Boys, S. F., and Handy, N. C., 1969, Proc. R. Soc. London Ser. A 310, 43.

[5] Boys, S. F., and Handy, N. C., 1969, Proc. R. Soc. London Ser. A 310, 63.

[6] Boys, S. F., and Handy, N. C., 1969, Proc. R. Soc. London Ser. A 311, 309.

[7] Handy, N. C., 1969, J. Chem. Phys. 51, 3205.

[8] Handy, N. C., 1971, Mol. Phys. 21, 817.

[9] Handy, N. C., 1972, Mol. Phys. 23, 1.

[10] Handy, N. C., 1973, J. Chem. Phys. 58, 279.

[11] Umrigar, C. J., Wilson, K. G., and Wilkins, J. W., 1988, Phys. Rev. Lett. 60, 1719.

[12] Schmidt, K. E., and Moskowitz, J. W., 1990, J. Chem. Phys. 93, 4172.

[13] Huang, C.-J., Umrigar, C. J., and Nightingale, M. P., 1997, J. Chem. Phys. 107, 3007.

[14] Drummond, N. D., Towler, M. D., and Needs, R. J., 2004, Phys. Rev. B 70, 235119. 
[15] Lin, X., Zhang, H., and Rappe, A. M., 2000, J. Chem. Phys. 112, 2650.

[16] Umrigar, C. J., Toulouse, J., Filippi, C., Sorella, S., and Hennig, R. G., 2007, Phy. Rev. Lett. 98, 110201.

[17] Luo, H., Hackbusch, W., and Flad, H.-J., 2009, J. Chem. Phys. 131, 104106.

[18] Krotscheck, E., 1984, Ann. Phys. (N.Y.) 155, 1.

[19] Krotscheck, E., 1985, Phys. Rev. B 31, 4267.

[20] Klopper, W., Manby, F. R., Ten-No, S., and Valeev, E. F., 2006, Int. Rev. in Phys. Chem. 25, 427.

[21] Ten-no, S., 2000, Chem. Phys. Lett. 330, 169.

[22] Ten-no, S., 2000, Chem. Phys. Lett. 330, 175.

[23] Hino, O., Tanimura, Y., and Ten-no, S., 2001, J. Chem. Phys. 115, 7865.

[24] Ten-no, S., and Hino, O., 2002, Int. J. Mol. Sci. 3, 459.

[25] Hino, O., Tanimura, Y., and Ten-no, S., 2002, Chem. Phys. Lett. 353, 317.

[26] Umezawa, N., and Tsuneyuki, S., 2003, Int. J. Quantum Chem. 91, 184.

[27] Umezawa, N., and Tsuneyuki, S., 2003, J. Chem. Phys. 119, 10015.

[28] Umezawa, N., and Tsuneyuki, S., 2004, J. Chem. Phys. 121, 7070.

[29] Umezawa, N., Tsuneyuki, S., Ohno, T., Shiraishi, K., and Chikyow, T., 2005, J. Chem. Phys. 122, 224101.

[30] Flad, H.-J., Hackbusch, W., Luo, H., and Kolb, D., 2005, Phys. Rev. B 71, 125115.

[31] Chinnamsetty, S. R., Luo, H., Hackbusch, W., and Flad, H.-J., (to be published).

[32] Huang, C.-J., Filippi, C., Umrigar, C. J., 1998, J. Chem. Phys. 108, 8838.

[33] Clementi, E., and Roetti, C., 1974, Atomic Data and Nuclear Data Tables 14, 177.

[34] Molpro is a package of ab initio programs written by Werner, H.-J., and Knowles, P. J., with contributions from Amos, R. D., Bernhardsson, A., Berning, A., Celani, P., Cooper, D. L., Deegan, M. J. O., Dobbyn, A. J., Eckert, F., Hampel, C., Hetzer, G., Korona, T., Lindh, R., Lloyd, A. W., McNicholas, S. J., Manby, F. R., Meyer, W., Mura, M. E., Nicklass, A., Palmieri, P., Pitzer, R., Rauhut, G., Schütz, M., Schumann, U., Stoll, H., Stone, A. J., Tarroni, R., and Thorsteinsson, T.

[35] Brown, M. D., Trail, J. R., Rios, P. L., and Needs, R. J., 2007, J. Chem. Phys. 126, 224110.

[36] Chakravorty, S. J., Gwaltney, S. R., Davidson, E. R., Parpia, F. A., and Fischer, C. F., 1993, Phys. Rev. A 47, 3649.

[37] Galek, P. T. A., Handy, N. C., and Lester Jr., W. A., 2006, Mol. Phys. 104, 3069. 